OPEN ACCESS

Edited by:

Chang-In Choi,

Pusan National University Hospital,

South Korea

Reviewed by:

Haruhiko Sugimura,

Hamamatsu University School of

Medicine, Japan

Jun-Hui Sun,

Zhejiang University, China

*Correspondence:

Jian-jun Han

jieruheweichuang@163.com;

handoctor@163.com

${ }^{\dagger}$ These authors have contributed equally to this work

Specialty section:

This article was submitted to Gastrointestinal Cancers,

a section of the journal

Frontiers in Oncology

Received: 14 June 2020 Accepted: 19 October 2020

Published: 15 December 2020

Citation:

Sun Y-d, Zhang H, Chen Y-q, Wu C-X, Zhang J-b, Xu H-r, Liu J-z and Han J-j (2020) HMGB1, the Next Predictor of

Transcatheter Arterial

Chemoembolization for Liver Metastasis of Colorectal Cancer?

Front. Oncol. 10:572418. doi: 10.3389/fonc.2020.572418

\section{HMGB1, the Next Predictor of Transcatheter Arterial Chemoembolization for Liver Metastasis of Colorectal Cancer?}

\author{
Yuan-dong Sun ${ }^{1 \dagger}$, Hao Zhang ${ }^{1 \dagger}$, Ye-qiang Chen ${ }^{2}$, Chun-xue Wu ${ }^{1,3}$, Jian-bo Zhang ${ }^{1}$, \\ Hui-rong $\mathrm{Xu}^{1}$, Jing-zhou Liu ${ }^{1}$ and Jian-jun Han ${ }^{1 *}$ \\ 1 Interventional Medicine Department, Shandong Cancer Hospital and Institute, Shandong First Medical University and Shandong \\ Academy of Medical Sciences, Ji'nan, China, ${ }^{2}$ Maternal and Child Health Care Hospital of Shandong Province, Ji'nan, China, \\ ${ }^{3}$ School of Medicine and Life Sciences, University of Ji'nan-Shandong Academy of Medical Sciences, Ji'nan, China
}

HMGB1 is an important mediator of inflammation during ischemia-reperfusion injury on organs. The serum expression of HMGB1 was increased significantly on the 1st day after TACE and decreased significantly which was lower on the 30th day after TACE. Tumor markers of post-DEB-TACE decreased significantly. The correlational analysis showed that patients with low HMGB1 expression had lower risks of fever and liver injury compared those with the higher expression, while the ORR is relatively worse. Patients with lower expression of HMGB1 had longer PFS, better efficacy, and higher quality of life. With the high post-expression, the low expression had lower incidence of fever and liver injury too. There was no statistical difference in the one-year survival among the different groups. The quality of life of all patients was improved significantly. The over-expression of HMGB1 in LMCRC is an adverse prognostic feature and a positive predictor of response to TACE.

Keywords: liver metastasis of colorectal cancer, drug-eluting beads transarterial chemoembolization, transarterial chemoembolization, high mobility group box 1, HMGB1

\section{HIGHLIGHTS}

The findings of this study show that patients with low expression of HMGB1 before TACE have a lower incidence of severe liver damage. Post-TACE liver damage is proportional to the pre-TACE expression level of HMGB1, and respondents who reported post-TACE lower levels of HMGB1 also reported significantly lower liver damage. The findings from these studies suggest that higher HMGB1 expression levels before TACE may be a prognosis of liver damage and efficacy. Taken together, these results exhibit that patients with severe HMGB1 changes after TACE had more severe liver damage and were less sensitive, but ORR and PFS of them were relatively better. These results confirm that the changes of HMGB1 maybe the predictor of liver damage and efficacy after TACE. 


\section{INTRODUCTION}

Colorectal cancer is one of the most common malignant tumors in the world, including colorectal cancer and rectal cancer (1). About $10-25 \%$ of colorectal cancer patients find simultaneous liver metastasis at the time of diagnosis $(2,3)$. When patients have distant metastasis outside the primary site, it is difficult to obtain satisfactory results only by surgical resection (4-6). For unresectable metastatic liver cancer, cryoablation, local thermal ablation of liver, transcatheter arterial infusion (TAI), proton therapy, liver radioactive particle implantation, and transcatheter arterial chemoembolization (TACE) are some good non-surgical treatment methods (7-10).

TACE is considered one of the most effective and safe treatment for advanced liver cancer (11). It is generally assumed to play a considerable role in liver solid tumors, but it has also been reported to have the potential to cause significant damage to liver function (12). There are three reasons that liver injury after TACE are common: the history of concomitant cirrhosis, chemotherapeutic drugs, and the process of ischemia-reperfusion in the liver (13). After TACE, the block of blood supply to local liver tissue at the embolic site leads to local ischemia and hypoxia. After a period of time (usually 7-30 days), local blood supply is restored under the dual action of flowing blood and the establishment of collateral circulation. Therefore, we can assume that the liver undergoes a complete ischemia-reperfusion process after TACE. Conventional TACE (c-TACE) and drug-eluting bead transcatheter aterial chemoembolization (DEB-TACE) are widely used at present. Lipiodol suspended with an anticancer drugs and gelatin sponge particles served as embolic-agents are widely used in c-TACE. Chemotherapeutic drugs were delivered to the tumor by superselective catheterization, and then the nutrient vessels were sealed with embolic materials. At present, several novel spherical embolic drugs-carrying/drug-eluting beads (DEBs) have been developed to release the drug slowly and long-term, reduce liver damage and improve the local concentration of anticancer drugs. The biggest difference between the two is that DEB-TACE combines drugs and embolic materials in drug-loaded microspheres, but their effects on local blood disruption in the liver are similar. Regardless of the difference of treatment modalities, some chemotherapeutic drugs (such as irinotecan, doxorubicin, oxaliplatin, etc.) inevitably have a killing effect on peritumoral tissue (14). Lead to the powerful killing effect of chemotherapeutics, normal liver tissues appeared damaged, necrotic, and apoptotic (14). On the other hand, some recent findings show that inflammatory mediators after TACE play a role in the reestablishment of collateral circulation. Therefore, after TACE, timely prediction and clinical treatment of patients' liver damage can effectively reduce the possibility of tragic outcomes. However, there are certain drawbacks of the current liver function test, like insufficient sensitivity and higher latency. When the results of the liver function test after TACE showed obvious abnormalities, patients often have reached the level of severe liver damage. It is necessary to find a critical demand for prognostic and predictive biomarkers in liver damage (15).

Previous studies in patients with primary liver cancer have shown that expression of high mobility group box-1 (HMGB1) in local liver tissues can rise dramatically in a few hours after
TACE (16). Significant changes in serum expression of HMGB1 could be detected at 12 to $24 \mathrm{~h}$ after TACE, and it could reach the highest level at 28 to $36 \mathrm{~h}$. Finally, HMGB1 gradually returned to the normal level within the following month (17). In view of the repeated traumatic examination of the liver that will bring certain risks of complication to patients, the concentration level of HMGB1 in the blood is the predictor to analyze the liver damage and avoid the bad impact of repeated liver biopsy. In this study, we studied the level of HMGB1 in the blood after TACE and verified the predictive ability of HMGB1 on liver damage and the efficacy of TACE. To this end, we generated a comprehensive review after TACE at the liver damage, safety and progression-free survival time (PFS) by blood samples, clinical information, and the results of follow-up.

\section{METHOD}

\section{Study Design}

A prospective, randomized study recruited $106 \mathrm{LMCC}$ patients from December 2017 to July 2019 in Shandong Tumor Hospital as previously described. All procedures were performed with a protocol approved by the ethics committee. Patients were required to be 18 years of age or older and have a diagnosis of liver metastases from colorectal cancer. Patients with a prior anticancer treatment within 2 months were not eligible for enrollment. Prior to the collection of biological samples and TACE, all patients were required to give full informed consent. All patients had radiologic imaging either by computed tomography scanning (CT) and/or magnetic resonance imaging (MRI) before TACE to document the presence of any other metastases. Serum tumor markers (CA19-9, CEA), serum HMGB1 level, liver function and blood cell cluster differentiation antigen were examined 4 to $6 \mathrm{~h}$ before TACE and 1, 3 , $5,7,30$ days after treatment. Laboratory analysis of serum preparation was performed at the Shandong Province Cancer Hospital Central Laboratory. Approximately $10 \mathrm{ml}$ of peripheral blood was drawn by the peripheral vein puncture in two standard serum tubes and centrifuged (10 min, 2,000g, room temperature) within $24 \mathrm{~h}$ following the collection time to remove clots. The researcher collected and dispensed the serum into multiple $2 \mathrm{ml}$ cryotubes and stored it at $-80^{\circ} \mathrm{C}$. Any contaminated samples were excluded from the analysis. The concentration of HMGB1 in serum was measured by the ELISA kit (Novus Biologicals, LLC, US) and immune cells were determined by the flow cytometry assay (Thermo Fisher Scientific Inc. US). All assays were run according to the manufacturer's instructions, and all controls were within the ranges provided by the manufacturer. In this study, all patients underwent TACE for the liver metastases and symptomatic treatment for the possible adverse reaction. Enhanced imaging examination obtained from all cases was centrally reviewed by two radiologists to verify the diagnoses made by the researcher. The patients had a regular review with their physician every month during the first six months and then every two months until the end of follow-up.

The treatments were performed by two designated interventional radiology physicians (20- and 11-years' experience). The medical 
imaging results were viewed by a radiologist (minimum 10 years of experience) and reviewed by another radiologist. The follow-up information and results were compiled and maintained by a designated researcher. Data analysis was conducted independently by two researchers.

\section{Group}

Due to the differences in drug release rate and local concentration between the two TACE modalities, we initially divided patients into DEB-TACE (CalliSpheres ${ }^{\circledR}$, Jiangsu Hengrui Medicine Co. Ltd., Jiangsu, P.R. China) group and c-TACE groups. Then, we classified that patients with preHMGB1 level in serum above $17.5 \mathrm{pg} / \mathrm{ml}$ as the preoperative high expression group and others as the preoperative low expression group. Whether the change of HMGB1 concentration in the sample on the first day after TACE is more than $50 \%$ is defined as the grouping standard. According to the high change group of HMGB1 before TACE increased by more than $50 \%$, and the patients with variation less than $50 \%$ were low change group.

\section{Follow-Up}

PFS, the most important efficacy indicator in this study, is the time between the date the patient enters the group for treatment and any documented tumor progression or death from any cause (not limited to death from cancer). The treatment outcomes of TACE can be classified as complete response (CR), partial response (PR), stable disease (SD), and progressive disease (PD) according to mRECIST1.1. The objective remission rate (ORR) in this study is the proportion of patients whose target tumor shrinks to the SD level and remains there for a period.

\section{Statistical Analysis}

The statistical data in this study were analyzed using SPSS version 22.0 (SPSS Inc., Chicago, US) and GraphPad Prism 8.0 (GraphPad Software Inc., San Diego, CA 92108). Sample size in this study was calculated by PASS 15.0 (NCSS, LLC. Kaysville, Utah, USA). The sample size was determined by power analysis using preliminary data obtained in our laboratory with the following assumptions: $\alpha$ of 0.05 (two-tailed), power of $90 \%$, difference in patients between before and after TACE, and a standard deviation of $17.5 \mathrm{pg} / \mathrm{L}$. Fisher's exact test (two-tailed) was used to compare categorical variables and Mann-Whitney U test (two-tailed) for continuous variables. PFS was analyzed using the Kaplan-Meier method and compared via the log-rank test. Comparisons were made using the log-rank test (for univariate analysis). Between-group comparisons were examined using either the t-test or the chi-square test. The correlation analysis was performed using Poisson's test, and p-values less than 0.05 were considered statistically significant. All tables are drawn by Microsoft Office Word 2019 (Microsoft, Redmond, WA, USA).

\section{RESULTS}

A total of 126 patients enrolled in the study, and 106 of them (82 males and 24 females) were evaluable. Patients were divided into two groups: 56 of them received DEB-TACE and the rest received c-TACE. The mean age of the evaluable study cohort was 61 years old (range: 30 to 88 ), with a mean age for the c-TACE group of 60 years old (range: 30 to 79) and DEB-TACE 62 years old (range: 38 to 88 ). There were 51 patients with rectal cancer (23 c-TACE and 28 DEB-TACE) and 55 patients with colon cancer (27c-TACE and 28 DEB-TACE) diagnosed in the study group. Patient demographics and characteristics are illustrated in Table 1. Data analysis was conducted independently by two researchers.

\section{Level of HMGB1 of Post-TACE}

The patients' average HMGB1 of pre-TACE was $19.68 \mathrm{pg} / \mathrm{ml}$ and at 1 st after TACE was $32.25 \mathrm{pg} / \mathrm{ml}, \mathrm{p}<0.05$. At 30 days after treatment, the level was $17.19 \mathrm{PG} / \mathrm{ml}$, which was statistically significant. The changes of HMGB1 expression in patients are shown in Table 1.

\section{Pre-TACE Level of HMGB1 and Prognosis}

The patients were grouped by the level of HMGB1 in the serum before TACE. The basic information about the four groups of patients is shown in Table 2.

A comparison of the changes in liver function during treatment in each group (Figure 1) revealed that most of the index markers failed to show sufficient statistical significance (Table 3), but the liver damage seems more severe in the high pre-TACE HMGB1 expression group, regardless of whether they received DEB-TACE or c-TACE.

TABLE 1 | Patients' characteristics before TACE.

\begin{tabular}{|c|c|c|}
\hline \multirow[t]{2}{*}{ Characteristics } & DEB-TACE & C-TACE \\
\hline & Patients & Patients \\
\hline \multicolumn{3}{|l|}{ Gender } \\
\hline Male & $41(73.21 \%)$ & $41(82.00 \%)$ \\
\hline Female & $15(26.79 \%)$ & $9(18.00 \%)$ \\
\hline \multicolumn{3}{|l|}{ Age } \\
\hline$<60$ & 22 (39.29\%) & $22(44.00 \%)$ \\
\hline$\geq 60$ & $34(60.71 \%)$ & $28(56.00 \%)$ \\
\hline \multicolumn{3}{|l|}{ ECOG Score ${ }^{a}$} \\
\hline 0 & $2(3.57 \%)$ & $6(12.00 \%)$ \\
\hline 1 & $28(50.00 \%)$ & $27(54.00 \%)$ \\
\hline 2 & $20(35.71 \%)$ & 12 (24.00\%) \\
\hline 3 & $6(10.72 \%)$ & $5(10.00 \%)$ \\
\hline \multicolumn{3}{|l|}{$B C L C{ }^{b}$} \\
\hline A & $12(21.43 \%)$ & $9(18.00 \%)$ \\
\hline B & $44(78.57 \%)$ & $41(82.00 \%)$ \\
\hline \multicolumn{3}{|l|}{ Tumor differentiation } \\
\hline No reported & 22 (39.29\%) & $20(40.00 \%)$ \\
\hline Low & $10(17.86 \%)$ & $6(12.00 \%)$ \\
\hline Moderate & $12(21.43 \%)$ & $10(20.00 \%)$ \\
\hline High & $12(21.43 \%)$ & $14(28.00 \%)$ \\
\hline \multicolumn{3}{|c|}{ The expression of HMGB1 (pg/ml) } \\
\hline Pre-TACE & $19.14 \pm 3.91$ & $19.98 \pm 3.98$ \\
\hline 1st after TACE & $31.55 \pm 7.15$ & $32.86 \pm 7.62$ \\
\hline 3rd after TACE & $31.31 \pm 7.10$ & $32.55 \pm 7.65$ \\
\hline 5th after TACE & $30.75 \pm 7.21$ & $32.08 \pm 7.52$ \\
\hline 7th after TACE & $28.78 \pm 6.69$ & $30.16 \pm 6.81$ \\
\hline 30th after TACE & $17.39 \pm 2.86$ & $17.01 \pm 2.44$ \\
\hline
\end{tabular}

${ }^{a}$ ECOG Score : Eastern Cooperative Oncology Group Score Standard.

${ }^{b}$ BCLC: Barcelona Clinic Liver Cancer. 


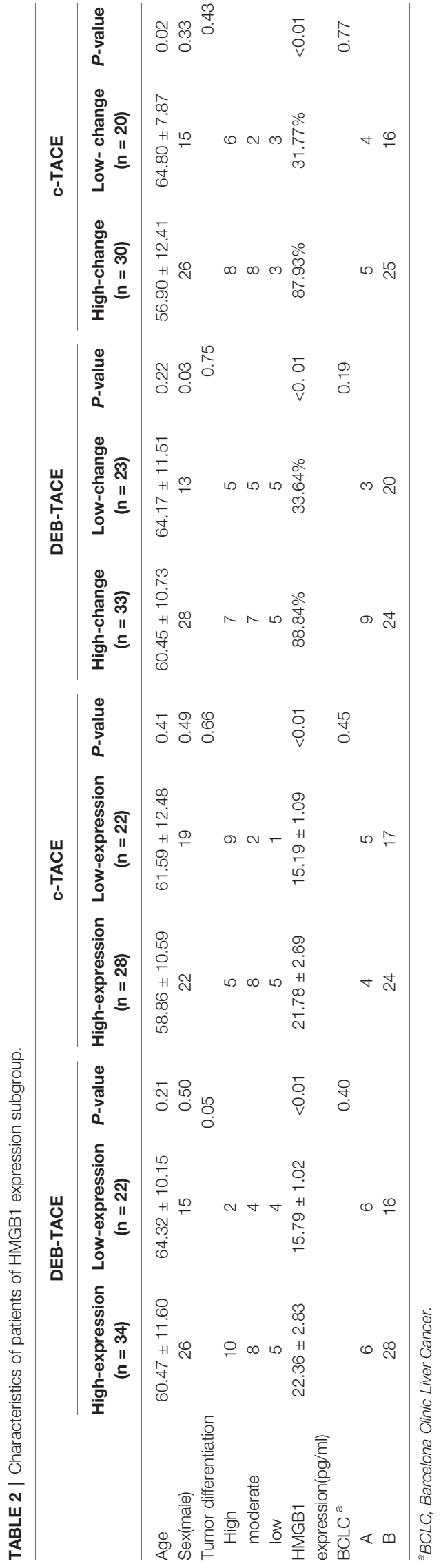

Figure 2 depicts the changes of tumor markers and immune function of patients during the treatment, which have more detailed comparisons in Table 3. There was a significant decrease of tumor marker in all patients after treatment. The patients in the four groups had transient immune disorders after TACE, but the degree of inhibition in the low expression group was slight than the others. Immune function of all could be recovered to the level of pre-treatment for one month.

The results of statistics of some common adverse reactions rate after TACE are shown in Table 4. Patients had similar risks of vomiting, abdominal pain, and nausea, but those who exhibited high expression of HMGB1 before TACE had a higher risk of fever.

The analysis of the treatment outcomes of the four groups of patients is shown in Table 4. Combined with the significant decrease of tumor markers, it can be found that most patients have a good treatment effect even if they receive different TACE. The table also shows that the quality of life of all patients was significantly improved after TACE.

As shown in Figure 3A, a correlation was found between the pre-expression of HMGB1 and PFS. The differences between the level of HMGB1 and 1-year survival are highlighted in Figure 3B.

\section{The Change of Post-TACE Level of HMGB1 and Prognosis}

The basic information about the four groups of patients is shown in Table 3.

From the information of Figures 4 and Table 5, we can find that there is a certain relation between the changes of liver function and the changes of HMGB1. The rise of HMGB1 is accompanied by subsequent liver function damage, which means that the rise of HMGB1 probably indicates the severity of the liver injury. It can be seen from the change trend chart that as the change curve of HMGB1 showed a significant rise, the markers of liver damage also showed a significant upward trend in the following days.

Table 5 shows the changes of tumor markers and immune function after TACE. The trend chart (Figure 5) shows that the degree of immunosuppression after DEB-TACE was slightly severer than that after c-TACE; however, the difference between them revealed no statistically significant differences.

The adverse reactions after the TACE of the four groups were analyzed, and the results are shown in Table 6. Fever is the most closely related adverse reaction to the change of HMGB1.

The treatment results of the four groups are shown in Table 6. The patients with a small increase in HMGB1 after TACE have a relatively good treatment effect. The PFS and one-year survival are shown in Figures 3C, D.

As can be seen from the data in Table 6, the quality of life of all patients benefits from treatment.

\section{DISCUSSION}

In this study, the level of HMGB1 was found significantly elevated in the blood after TACE. It expands the knowledge on the association between HMGB1 and treatment outcome in LMCRC by showing its magnitude rather than just showing that there is a statistically 
ALT

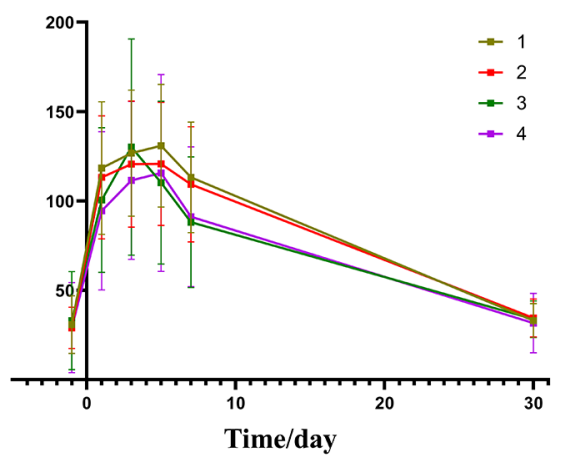

ALB

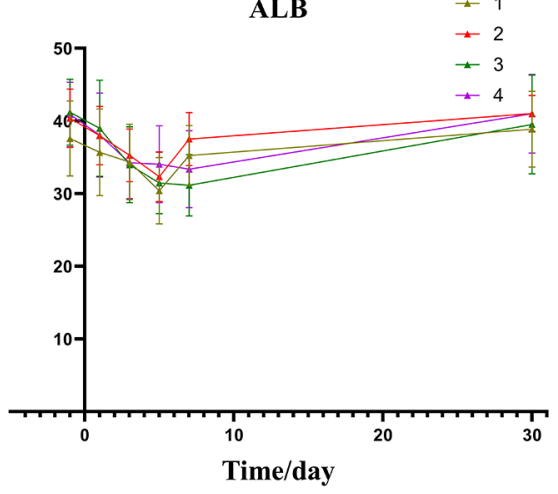

ALP

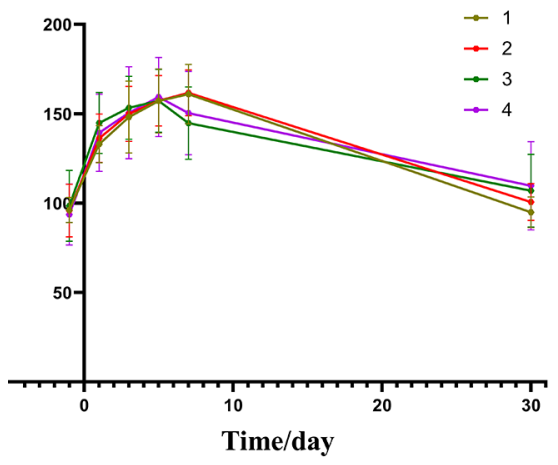

AST

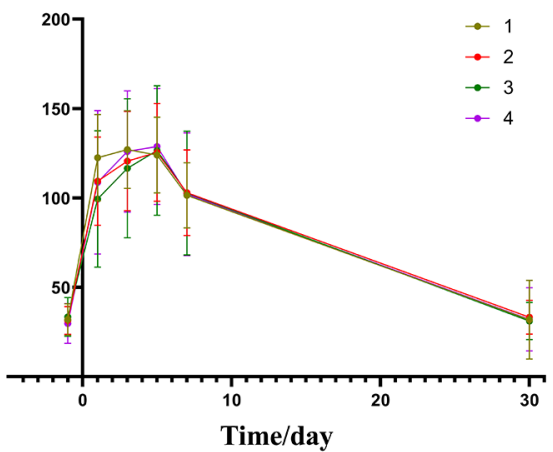

TBIL

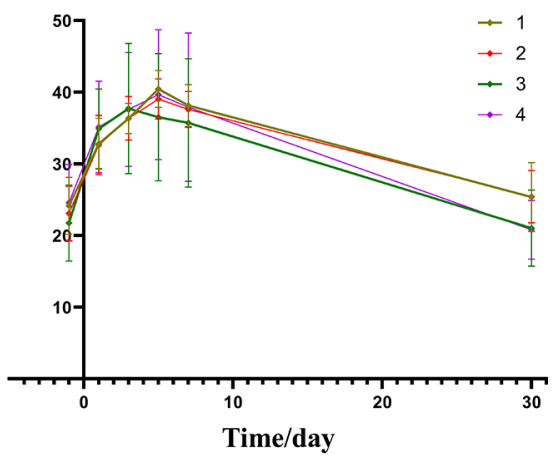

HMGB1

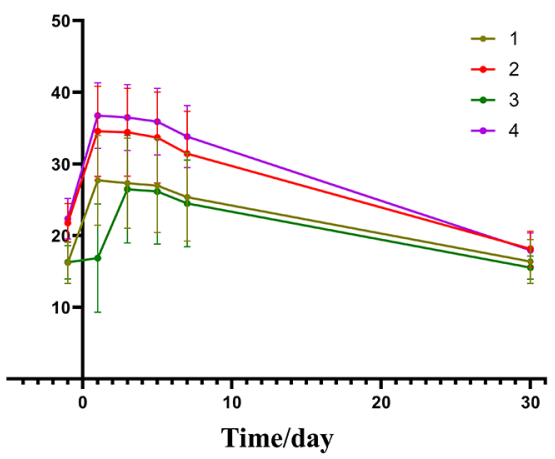

FIGURE 1 | Group1: low pre-expression of HMGB1 with c-TACE; Group2: high pre-expression of HMGB1 with c-TACE; Group3: low pre-expression of HMGB1 with DEB-TACE; Group4: high pre-expression of HMGB1 with DEB-TACE.

significant relationship. HMGB1 was more enriched in the serum of patients with severe liver impairment compared to the preoperative low-expression group. We identified a highly significant relation among HMGB1expression, liver damage, and PFS.

In addition, the analysis of the changes of HMGB1 after TACE can improve the sensitivity of it in the diagnosis of liver function damage. HMGB1 may be a possible prognostic factor for adverse reactions in patients with LMCRC.

Due to their stability and specificity in most bodily fluids, HMGB1 provides a high potential to serve as a liquid biopsy tool for some cancers and sterile inflammation (18). Some researchers performed proteomic analyses to the clinical significance of HMGB1 in serum- purified exosomes from malignant mesothelioma cancer patients and identified it as potential biomarkers in diagnosis and prognosis (19, 20). Dr. Venereau believes that high levels of serum hyper-acetylated HMGB1 are sensitive disease biomarkers (21). He also found that injection of HMGB1 accelerates tissue repair by acting on muscle stem cells, hepatocytes, and infiltrating cells (22). Dr. Liu concludes that HMGB1 protein is a valuable marker for the progression of CRC patients. High HMGB1 expression is associated with poor overall survival in patients with CRC (23).

In the case of LMCRC, we have further confirmed strong correlations between elevated expression of HMGB1 and liver damage or PFS and discovered strong correlations between 
DEB-TACE

C-TACE

High HMGB1 expression Low HMGB1 expression $p$-value

\section{$95 \% \mathrm{Cl}$}

High HMGB1 expression Low HMGB1 expression p-value

$95 \% \mathrm{Cl}$

Pre-ALT

1st post-ALT

3rd post-ALT

5th post-ALT

7th post-ALT

30th post-ALT

Pre-AST

1st post-AST

3rd post-AST

5th post-AST

7th post-AST

30th post-AST

Pre-ALB

1st post-ALB

3rd post-ALB

5th post-ALB

7th post-ALB

30th post-ALB

Pre-TBIL

1st post-TBIL

3rd post-TBIL

5th post-TBIL

7th post-TBIL

30th post-TBIL

Pre-ALP

1st post-ALP

3rd post-ALP

5th post-ALP

7th post-ALP

30th post-ALP

Pre-CEA

30th post-CEA

Pre-CA19-9

30th post- CA19-9

Pre-CD3+

7th post-CD3+

30th post-CD3+

Pre-CD19+

7th post-CD19+

30th post-CD19+

Pre-NK cell

7th post-NK cell

30th post-NK cell

Pre-CD3+CD4+

7th post-CD3+CD4+

30th post-CD3+CD4+
$29.14 \pm 25.26$

$94.48 \pm 44.22$

$111.59 \pm 44.25$

$115.69 \pm 55.04$

$91.17 \pm 39.10$

$31.74 \pm 16.61$

$29.80 \pm 11.08$

$108.78 \pm 40.11$

$126.04 \pm 33.92$

$128.75 \pm 32.42$

$102.14 \pm 34.33$

$32.10 \pm 17.73$

$40.89 \pm 4.43$

$38.07 \pm 5.78$

$34.26 \pm 4.95$

$34.05 \pm 5.29$

$33.36 \pm 5.31$

$41.00 \pm 5.41$

$24.57 \pm 5.34$

$35.14 \pm 6.39$

$37.61 \pm 7.97$

$39.66 \pm 9.07$

$37.92 \pm 10.35$

$20.81 \pm 4.10$

$93.63 \pm 17.09$

$139.34 \pm 21.65$

$150.61 \pm 25.76$

$159.43 \pm 22.13$

$150.40 \pm 23.28$

$109.69 \pm 24.76$

$547.90 \pm 1,506.18$

$416.60 \pm 1,120.93$

$547.39 \pm 1,777.83$

$137.11 \pm 234.16$

$64.45 \pm 7.46$

$56.42 \pm 5.21$

$66.02 \pm 7.10$

$11.55 \pm 3.51$

$10.19 \pm 3.15$

$11.94 \pm 3.36$

$24.89 \pm 8.79$

$20.18 \pm 7.69$

$24.93 \pm 8.76$

$39.67 \pm 6.70$

$43.10 \pm 6.31$

$39.95 \pm 7.37$
$33.12+27.48$

$100.57 \pm 40.44$

$130.13 \pm 60.53$

$110.23 \pm 45.58$

$88.15 \pm 36.50$

$33.94 \pm 10.18$

$33.49 \pm 10.87$

$99.49 \pm 38.18$

$116.64 \pm 38.83$

$126.58 \pm 36.26$

$102.74 \pm 34.61$

$31.23 \pm 10.43$

$41.22 \pm 4.54$

$39.00 \pm 6.61$

$33.98 \pm 5.22$

$31.46 \pm 4.22$

$31.17 \pm 4.23$

$39.53 \pm 6.82$

$21.76 \pm 5.33$

$34.91 \pm 5.56$

$37.73 \pm 9.09$

$36.52 \pm 8.89$

$35.72 \pm 8.96$

$21.05 \pm 5.32$

$98.52 \pm 19.79$

$144.91 \pm 17.02$

$153.41 \pm 17.67$

$157.26 \pm 17.56$

$144.74 \pm 20.31$

$106.94 \pm 20.36$

$369.10 \pm 646.92$

$247.60 \pm 409.36$

$416.60 \pm 1120.93$

$286.40 \pm 712.42$

$66.94 \pm 9.76$

$57.09 \pm 5.24$

$67.68 \pm 8.93$

$11.48+3.90$

$10.13 \pm 3.41$

$11.94 \pm 3.83$

$27.03 \pm 8.04$

$22.54 \pm 7.92$

$27.50 \pm 7.98$

$37.24 \pm 5.87$

$40.55 \pm 7.22$

$38.11 \pm 7.78$
3.97(-10.37-18.31)

6.09(-17.39-29.56)

18.53(-9.55-46.62)

$-5.47(-33.75-22.82)$

$-3.02(-23.93-17.88)$

2.20(-5.73-10.13)

3.70(-2.34-9.73)

$-9.30(-30.89-12.30)$

$-9.40(-29.10-10.30)$

$-2.17(-20.80-16.64)$

0.60(-18.29-19.49)

$-0.87(-8.42-6.69)$

$0.34(-2.12-2.79)$

$0.93(-2.42-4.28)$

$-0.28(-3.05-2.50)$

$-2.59(-5.28-0.10)$

$-2.19(-4.89-0.51)$

$-1.46(-4.75-1.83)$

$-2.82(-5.75-0.11)$

$-0.23(-3.57-3.10)$

$0.12(-4.50-4.74)$

$-3.14(-8.08-1.79)$

$-2.20(-7.60-3.19)$

$0.24(-2.30-2.77)$

$4.89(-4.43-14.20)$

$5.58(-5.38-16.54)$

2.79(-8.85-14.44)

$-2.18(-13.41-9.05)$

$-5.66(-17.82-6.51)$

$-2.75(-15.44-9.95)$

$-178.80(-861.57-503.97)$

20.63(-204.03-245.29)

$-130.78(-984.19-722.62)$

149.28(-174.99-473.56)

2.49(-2.13-7.11)

$0.66(-2.20-3.53)$

$1.66(-2.66-5.97)$

$-0.07(-2.09-1.84)$

$-0.06(-1.85-1.72)$

$-0.01(-1.95-1.95)$

2.14(-2.53-6.81)

$2.37(-1.90-6.63)$

$2.57(-2.07-7.21)$

$-2.43(-5.94-1.07)$

$-2.55(-6.21-1.12)$

$-1.85(-5.98-2.29)$
$29.07 \pm 11.55$

$113.21 \pm 34.44$

$120.67 \pm 35.33$

$120.77 \pm 34.39$

$109.31 \pm 32.20$

$34.57 \pm 10.60$

$31.54+7.73$

$109.40 \pm 24.73$

$120.69 \pm 27.76$

$125.54 \pm 27.37$

$103.01 \pm 23.99$

$33.33 \pm 9.46$

$40.37 \pm 4.01$

$37.97 \pm 4.01$

$35.28 \pm 3.62$

$32.33+3.40$

$41.04 \pm 2.49$

$23.10 \pm 3.77$

$32.64 \pm 4.12$

$36.37 \pm 3.06$

$39.05 \pm 2.79$

$37.61 \pm 2.51$

$25.42 \pm 3.66$

$95.84 \pm 14.74$

$136.35 \pm 13.60$

$149.94+15.35$

$157.31 \pm 14.06$

$161.76 \pm 12.85$

$100.65 \pm 10.29$

$433.67 \pm 444.94$

$204.43 \pm 206.56$

$565.02 \pm 890.67$

$330.32 \pm 567.47$

$64.65 \pm 13.57$

$58.43 \pm 5.59$

$67.58 \pm 8.78$

$11.44+4.05$

$10.05 \pm 3.34$

$11.75 \pm 3.89$

$25.41 \pm 9.34$

$19.43+7.68$

$24.65 \pm 9.16$

$36.47 \pm 6.77$

$41.94 \pm 6.54$

$36.16 \pm 6.78$

$30.82 \pm 16.12$

$118.42 \pm 37.04$

$126.74 \pm 35.32$

$130.92 \pm 34.28$

$113.28 \pm 30.96$

$33.07 \pm 9.56$

$32.08+8.88$

$122.55 \pm 24.09$

$127.07 \pm 21.70$

$124.07 \pm 21.24$

$101.51 \pm 18.19$

$31.94 \pm 22.00$

$37.60 \pm 5.17$

$35.70 \pm 5.97$

$34.36 \pm 5.19$

$30.41+4.57$

$35.24 \pm 4.11$

$38.87 \pm 5.22$

$24.15 \pm 3.97$

$32.83 \pm 3.54$

$36.32 \pm 2.12$

$40.46 \pm 2.57$

$38.16 \pm 2.91$

$25.36 \pm 4.81$

$96.20 \pm 6.99$

$133.06 \pm 10.40$

$148.18 \pm 20.17$

$157.13 \pm 17.90$

$161.03 \pm 16.70$

$94.91 \pm 8.61$

$542.61 \pm 1139.32$

$214.52+318.59$

$592.34+1437.19$

$161.34 \pm 231.36$

$65.42 \pm 8.63$

$58.87 \pm 5.09$

$66.40 \pm 7.30$

$9.88+2.41$

$8.31 \pm 2.25$

$10.15 \pm 2.44$

$25.38 \pm 8.20$

$20.21 \pm 7.14$

$25.55+7.98$

$39.07 \pm 8.56$

$44.24 \pm 6.50$

$39.04 \pm 8.99$ 


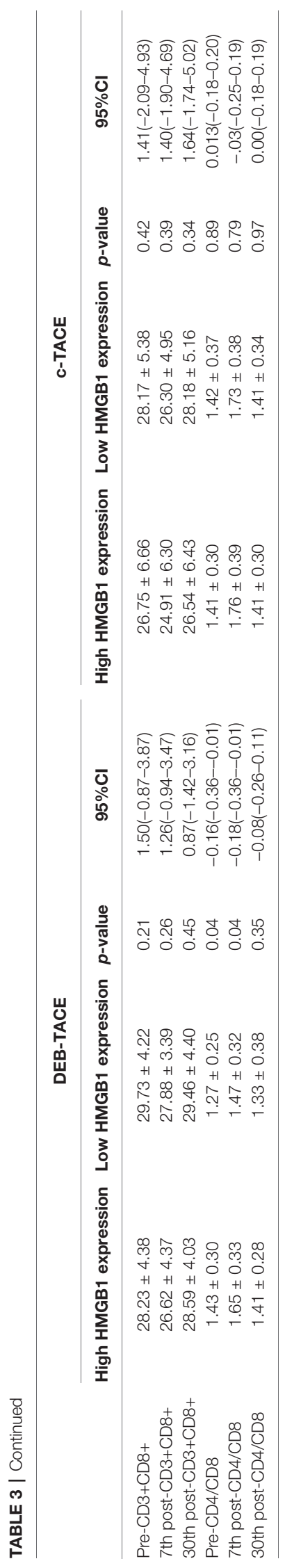

elevated the HMGB1 and the effect of palliative treatment including the c-TACE and DEB-TACE.

This study focused on the expression and changes of HMGB1 in the serum of colorectal cancer patients with liver metastasis before and after TACE, and we explored the possibility of predicting liver injury, adverse reaction, and PFS after TACE by monitoring the changes of HMGB1. In this study, we detected and compared the expression of HMGB1 before TACE and on the first, third, fifth, and seventh days after TACE. The results of data analysis and change trend chart showed that the expression of HMGB1 changed significantly and reached the peak on the 1st day after TACE, and the expression was stable on the 3rd, 5th, and 7th days. The expression of HMGB1 returned to pre-TACE until the 30th day. For those with low expression of HMGB1 before TACE, the liver injury was slighter, while for those with the severe rise of HMGB1 after treatment, the liver injury was more serious. The expression level of HMGB1 was positively correlated with the degree of liver injury. This result suggests that the level of HMGB1 on the 1st day after treatment compared with pre-TACE expression may predict liver injury.

Embolization and reperfusion after TACE are a standard process of liver ischemia-reperfusion injury in patients (13). After complete embolization of the target vessel by the doctor, the tumor focus of the liver and the surrounding normal liver tissue will form a temporary ischemic area (13). The interruption of the blood supply has left the area in a state of ischemia, hypoxia, and nutrient deprivation, with a large number of tumor cells and hepatocyte death (24). However, the thrombolytic effect of TACE gradually declined with the subsequent constant flushing of blood from the ischemic area. At the same time, collateral circulation was established in the embolized area, which made the embolized area get blood perfusion again. In the whole process, the embolization of drugs and blood vessels not only killed tumor cell, but also produced liver damage. Treatment stress and the changes of tumor microenvironment can aggravate the acute liver failure (25).

HMGB1 plays an important role in the process of ischemiareperfusion after TACE, and its acetylation and release are mainly regulated by four main modes (18). Firstly, large amounts of hypoxanthine accumulated converted to xanthine during anaerobic respiration after the interruption of blood supply, and the resulting ROS prompt cells to release acetylated HMGB1 (26, 27). Secondly, Kupffer cells can be activated to release IL-1 $\beta$, IL-6, and TNF- $\alpha$, which can promote the acetylation of $\operatorname{HMGB1}(28,29)$. Thirdly, during the late stage of ischemia/reperfusion (the phase of injury caused by blood reperfusion), activated neutrophils and macrophages begin to converge and accumulate towards the ischemic area and stimulate HMGB1 release through the release of inflammatory factors. Lastly, ischemia-reperfusion can lead to $\mathrm{Ca}^{+}$overload, which caused abnormal mitochondrial membrane permeability transporter pore and abnormal electron transport in the respiratory chain to produce ROS, then stimulates the local massive release of HMGB1 $(30,31)$. Under the combined action of various factors, a large amount of acetylated HMGB1 is released into the extracellular space, and its expression in the ischemic area and body blood rises rapidly. After TACE, the blood vessels of the tumor and surrounding tissue are embolized, which causes 

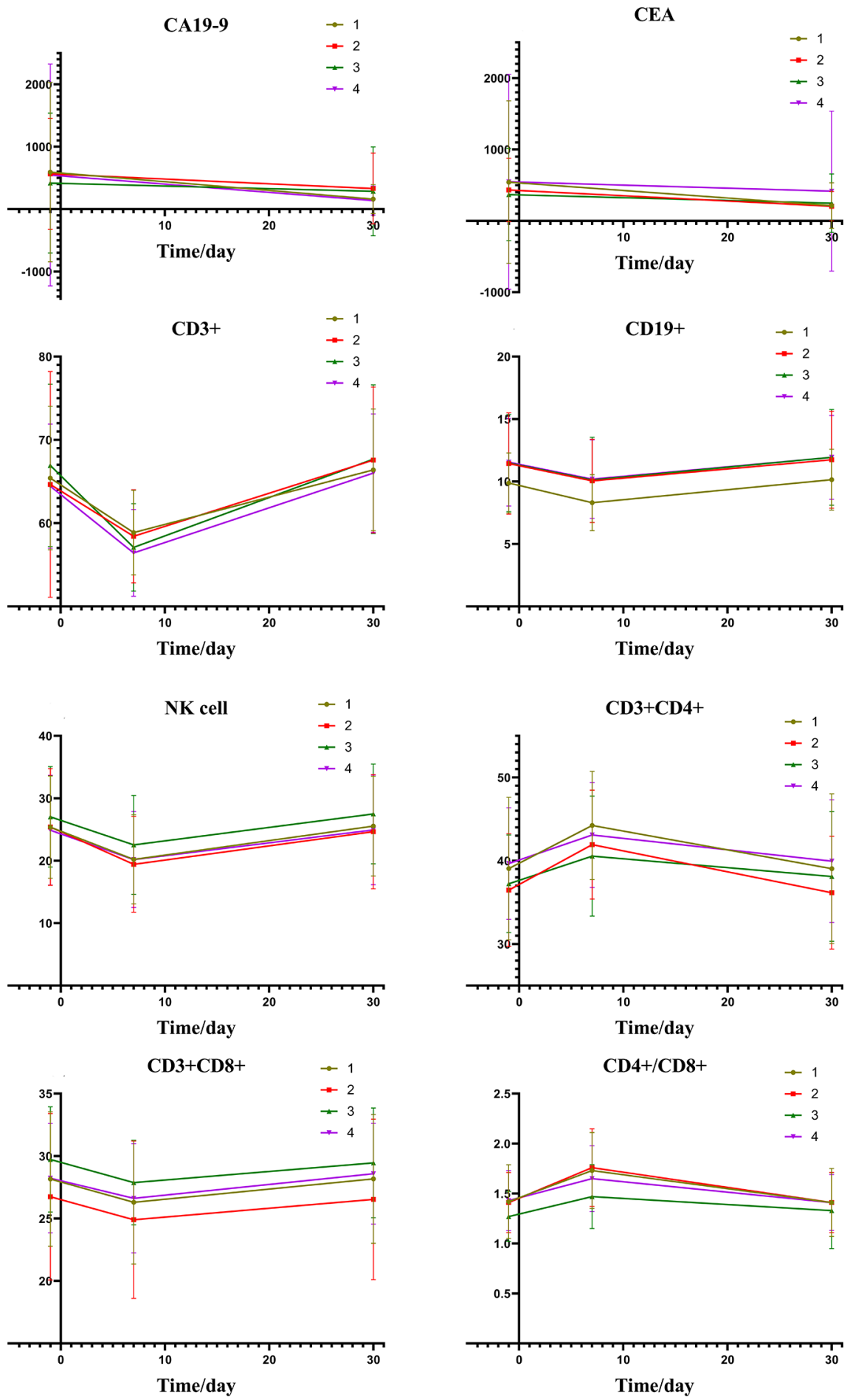

FIGURE 2 | Group1: low pre-expression of HMGB1 with c-TACE; Group2: high pre-expression of HMGB1 with c-TACE; Group3: low pre-expression of HMGB1 with DEB-TACE; Group4: high pre-expression of HMGB1 with DEB-TACE. 
TABLE 4 | Expression of HMGB1 group, common adverse reactions treatment outcomes.

\begin{tabular}{|c|c|c|c|c|c|c|}
\hline & \multicolumn{3}{|c|}{ DEB-TACE } & \multicolumn{3}{|c|}{ C-TACE } \\
\hline & $\begin{array}{l}\text { High HMGB1 expression } \\
\text { (34) }\end{array}$ & $\begin{array}{l}\text { Low HMGB1 expression } \\
\text { (22) }\end{array}$ & $p$-value & $\begin{array}{l}\text { High HMGB1 expression } \\
\text { (28) }\end{array}$ & $\begin{array}{l}\text { Low HMGB1 expression } \\
\text { (22) }\end{array}$ & $p$-value \\
\hline fever & 19 & 5 & 0.01 & 20 & 9 & 0.03 \\
\hline vomit & 15 & 6 & 0.20 & 11 & 5 & 0.22 \\
\hline nausea & 20 & 9 & 0.19 & 15 & 8 & 0.23 \\
\hline abdominal pain & 17 & 7 & 0.19 & 12 & 6 & 0.26 \\
\hline hepatic failure & 0 & 0 & 0 & 0 & 0 & 0 \\
\hline $\mathrm{CR}^{\mathrm{a}}$ & 2 & 4 & & 1 & 3 & \\
\hline$P R^{b}$ & 16 & 14 & & 14 & 15 & \\
\hline $\mathrm{SD}^{\mathrm{c}}$ & 14 & 3 & & 10 & 4 & \\
\hline$P D^{d}$ & 2 & 1 & & 3 & 0 & \\
\hline $\mathrm{ORR}^{\mathrm{e}}$ & 18 & 18 & 0.02 & 15 & 18 & 0.03 \\
\hline Pre-score of Qol ${ }^{f}$ & 32.76 & 33.10 & & 31.72 & 20.91 & \\
\hline $\begin{array}{l}\text { Post-score of } \\
\text { Qol }\end{array}$ & 47.91 & 48.32 & & 48.29 & 47.86 & \\
\hline
\end{tabular}

${ }^{a} \mathrm{CR}$, complete response.

${ }^{b} \mathrm{PR}$, partial response.

${ }^{\circ} S D$, stable disease.

${ }^{d} P D$, progressive disease.

${ }^{e} \mathrm{ORR}$, Objective response rate; $\mathrm{ORR}=\mathrm{CR}+\mathrm{PR}$.

${ }^{f} \mathrm{Qol}$, quality of life.

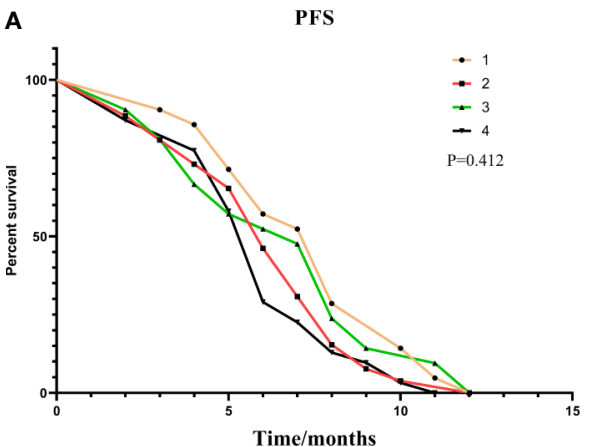

Time/months

B

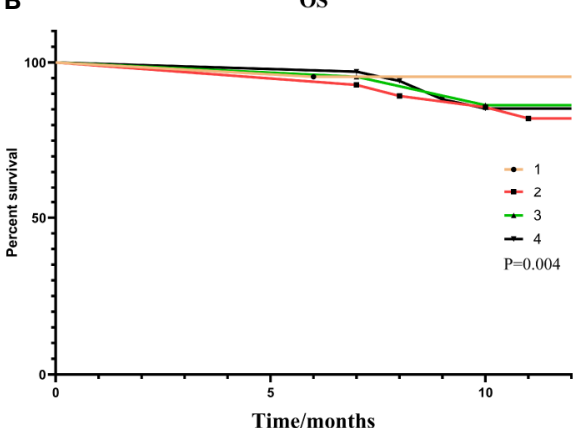

C

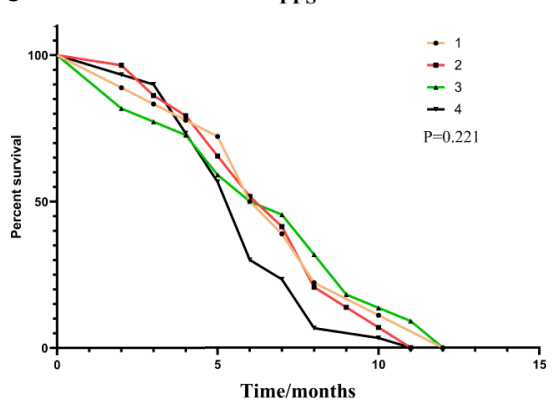

OS

D

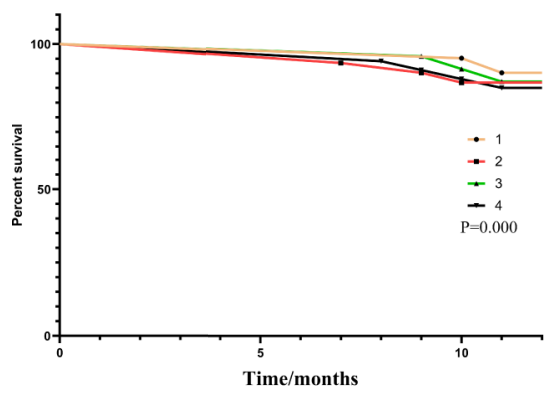

FIGURE 3 | (A, B) were subgroup analyses of HMGB1 expression before TACE, and (C, D) were subgroup analyses based on HMGB1 changes after TACE. In (A, B): Group1: low pre-expression of HMGB1 with c-TACE; Group2: high pre-expression of HMGB1 with c-TACE; Group3: low pre-expression of HMGB1 with DEB-TACE; Group4: high pre-expression of HMGB1 with DEB-TACE. In (C, D):Group1: low post-expression of HMGB1 with c-TACE; Group2: high postexpressions of HMGB1 with c-TACE; Group3: low post-expressions of HMGB1 with DEB-TACE; Group4: high post-expression of HMGB1 with DEB-TACE. 
ALT

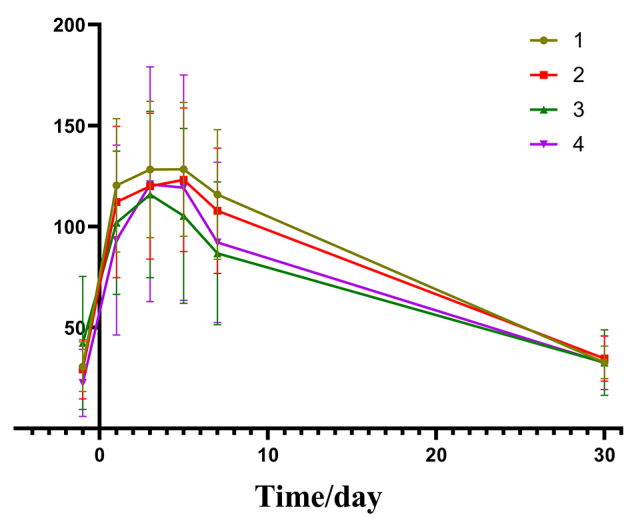

ALB

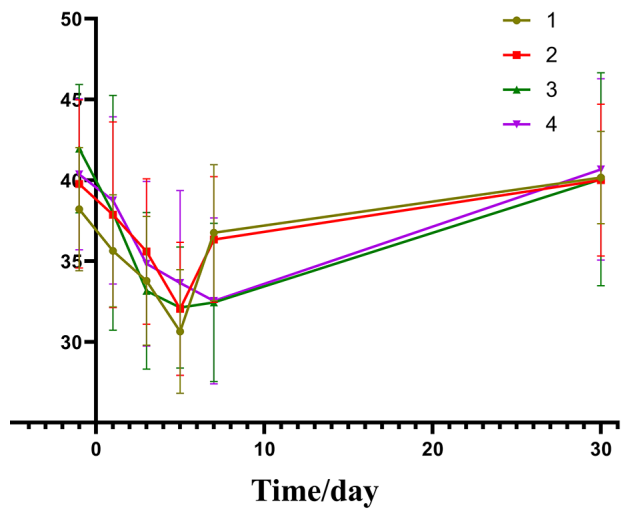

ALP

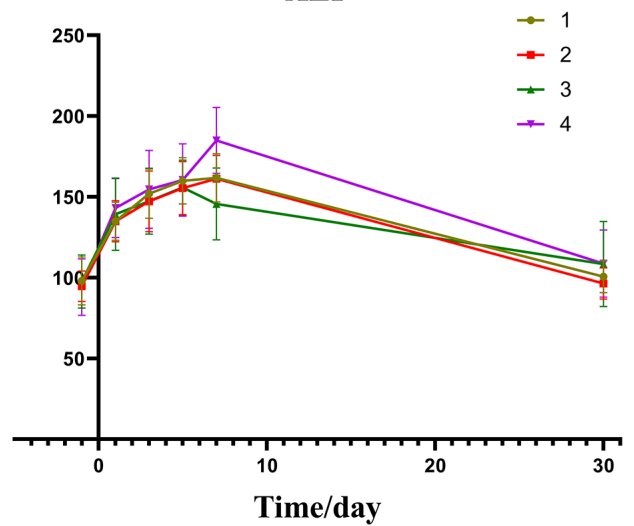

AST

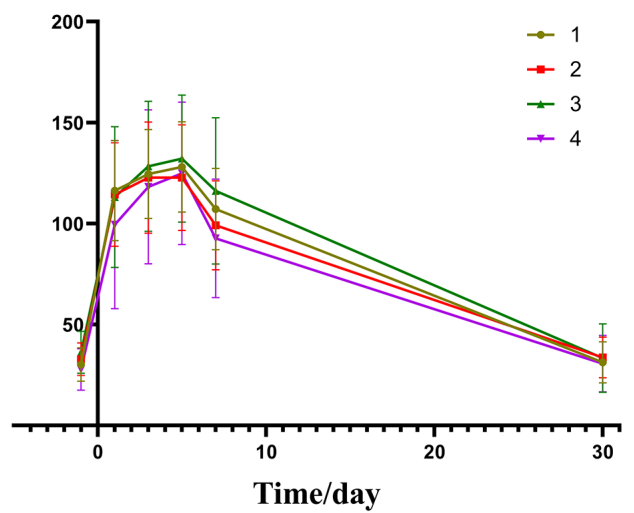

TBIL

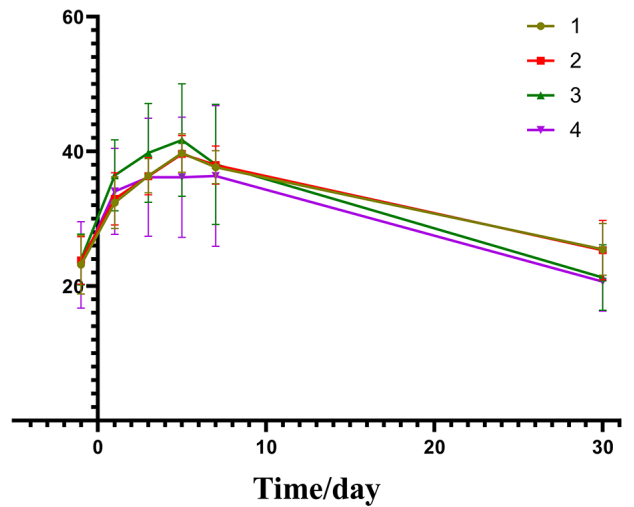

HMGB1

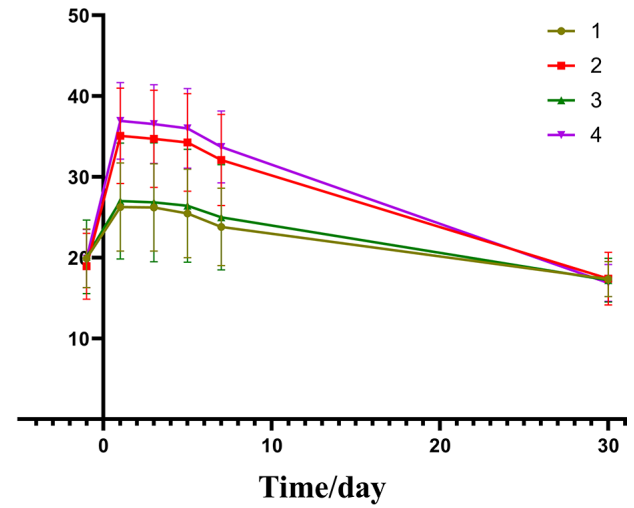

FIGURE 4 | Group1: low post-expression of HMGB1 with c-TACE; Group2: high post-expression of HMGB1 with c-TACE; Group3: low post-expression of HMGB1 with DEB-TACE; Group4: high post-expression of HMGB1 with DEB-TACE.

ischemia. However, the embolic material in the blood vessel is rinsed off by the blood, and the rapid formation of collateral vessels makes the ischemic area quickly regain blood supply. The large amount of HMGB1 produced in this process not only increased in the ischemic area but also reached the whole liver and the whole body by means of blood circulation, which leads to local and systemic inflammation of the liver (32). During ischemiareperfusion in the liver, HMGB1 plays an important mediating role: within $1-\mathrm{h}$ after TACE, cells in the ischemic area begin to necrotize and rupture, and release HMGB1. Induced by extracellular HMGB1 and other inflammatory factors, it leads to the release of more HMGB1 from the cells in the non-embolized area and mediates severe inflammatory response in the ischemic area $(31,33)$. Meanwhile, HMGB1 acetylated during the reperfusion phase can stimulate aggregated macrophages and monocytes to actively acetylate and release more HMGB1 (34). 
DEB-TACE

c-TACE

High-change $(\mathrm{n}=33) \quad$ Low-change $(\mathrm{n}=23) \quad p$-value

$95 \% \mathrm{Cl}$

High-change $(n=30) \quad$ Low-change $(n=20)$

$95 \% \mathrm{Cl}$

Pre-ALT

1st post-ALT

3rd post-ALT

5th post-ALT

7th post-ALT

30th post-ALT

Pre-AST

1st post-AST

3rd post-AST

5th post-AST

7th post-AST

30th post-AST

Pre-ALB

1st post-ALB

3rd post-ALB

5th post-ALB

7th post-ALB

30th post-ALB

Pre-TBIL

1st post-TBIL

post-TBIL

5th post-TBIL

7th post-TBIL

30th post-TBIL

Pre-ALP

1st post-ALP

3rd post-ALP

5th post-ALP

7th post-ALP

30th post-ALP

Pre-CEA

30th post-CEA

Pre-CA19-9

30th post- CA19-9

Pre-CD3+

7th post-CD3+

30th post-CD3+

Pre-CD19+

7th post-CD19+

30th post-CD19+

Pre-NK cell

7th post-NK cell

30th post-NK cell

Pre-CD3+CD4+

7th post-CD3+CD4+

30th post-CD3+CD4+
$22.57+16.67$

$93.37 \pm 47.00$

$120.93 \pm 58.19$

$119.32 \pm 55.90$

$92.20 \pm 39.77$

$27.75 \pm 10.27$

$99.52 \pm 41.65$

$118.17 \pm 38.13$

$124.91 \pm 35.29$

$92.71 \pm 29.33$

$30.60 \pm 14.00$

$40.37 \pm 4.68$

$38.76 \pm 5.18$

$34.84 \pm 5.10$

$33.65 \pm 5.72$

$32.54 \pm 5.14$

$40.67 \pm 5.62$

$23.14 \pm 6.42$

$34.07 \pm 6.38$

$36.17 \pm 8.78$

$36.15 \pm 8.93$

$36.35 \pm 10.44$

$20.65 \pm 4.41$

$94.15 \pm 17.51$

$143.07 \pm 18.30$

$154.71 \pm 24.12$

$160.50 \pm 22.40$

$184.98 \pm 204.77$

$108.72 \pm 20.79$

$605.42 \pm 1539.29$

$263.92 \pm 472.67$

$569.49 \pm 1806.03$

$160.49 \pm 253.45$

$65.77 \pm 9.25$

$56.30 \pm 4.72$

$66.64 \pm 8.39$

$11.72 \pm 3.71$

$10.32 \pm 3.26$

$12.13 \pm 3.62$

$25.72 \pm 8.83$

$20.92+7.88$

$25.95+8.75$

$38.23 \pm 6.50$

$42.00 \pm 6.69$

$38.47 \pm 7.26$
$42.38 \pm 32.99$

$101.89+35.49$

$115.92 \pm 41.25$

$105.26 \pm 43.34$

$86.80 \pm 35.37$

$32.69 \pm 16.20$

$36.26 \pm 10.36$

$113.17 \pm 34.92$

$128.34 \pm 32.24$

$132.18 \pm 31.47$

$16.25 \pm 36.31$

$33.42 \pm 16.91$

$41.96 \pm 3.97$

$37.99 \pm 7.27$

$33.16 \pm 4.84$

$32.13 \pm 3.75$

$32.45 \pm 4.90$

$40.07 \pm 6.59$

$23.94 \pm 3.80$

$36.46 \pm 5.30$

$39.79 \pm 7.34$

$41.70 \pm 8.36$

$38.07 \pm 8.93$

$21.27 \pm 4.88$

$97.56 \pm 16.39$

$139.31 \pm 22.43$

$147.41 \pm 20.49$

$155.82 \pm 16.97$

$145.59 \pm 22.23$

$108.44 \pm 26.29$

$294.36 \pm 561.91$

$193.69 \pm 289.73$

$390.58 \pm 1089.37$

$246.37 \pm 694.45$

$64.94 \pm 7.30$

$57.24 \pm 5.85$

$66.72 \pm 7.14$

$11.24 \pm 3.60$

$9.94 \pm 3.24$

$11.66 \pm 3.44$

$25.75 \pm 8.20$

$21.37 \pm 7.85$

$25.93 \pm 8.28$

$39.41 \pm 6.43$

$42.24 \pm 6.95$

$40.31 \pm 7.91$
19.82(4.68-34.95)

8.52(-13.60-30.64)

$-5.01(-33.30-23.29)$

$-14.05(-41.91-13.81)$

$-5.40(-26.12-15.32)$

$0.15(-7.75-8.04)$

8.51(2.90-14.12)

13.65(-7.62-34.91)

10.17(-9.35-29.69)

7.27(-11.13-25.67)

23.54(5.92-41.16)

2.82(-5.48-11.13)

$1.59(-0.81-3.99)$

$-0.77(-4.10-2.56)$

$-1.68(-4.40-1.04)$

$-1.52(-4.25-1.21)$

$-0.09(-2.84-2.65)$

$-0.60(-3.89-2.68)$

$0.80(-1.95-3.54)$

$2.39(-0.85-5.64)$

3.62(-0.86-8.09)

$5.54(0.80-10.28)$

$1.71(-3.65-7.08)$

0.62(-1.89-3.13)

$3.40(-5.89-12.69)$

$-3.76(-14.69-7.18)$

$-7.29(-19.66-5.07)$

$-4.68(-15.77-6.42)$

$-39.40(-125.59-46.80)$

$-0.28(-12.91-12.34)$

$-311.28(-985.27-363.15)$

70.23(-292.51-152.04)

$-178.90(-1025.43-667.63)$

85.89(-177.85-349.62)

$-0.83(-5.47-3.80)$

$0.94(-1.90-3.77)$

$0.08(-4.23-4.38)$

$-0.47(-2.47-1.52)$

$-0.38(-2.15-1.39)$

$-0.47(-2.41-1.46)$

$0.03(-4.64-4.70)$

$0.45(-3.84-4.73)$

$-0.03(-4.69-4.63)$

$1.17(-2.36-4.70)$

$0.24(-3.46-3.95)$

$1.85(-2.26-5.95)$
$29.28 \pm 14.62$

$112.22 \pm 37.52$

$120.06 \pm 36.13$

$123.15 \pm 35.59$

$107.82 \pm 31.02$

$34.65+11.30$

$32.84+8.07$

$114.42 \pm 25.65$

$122.80 \pm 27.52$

$122.78 \pm 26.18$

$99.11 \pm 22.03$

$33.67 \pm 10.08$

$39.78 \pm 5.20$

$37.87 \pm 5.75$

$35.60 \pm 4.51$

$32.05 \pm 4.12$

$36.34 \pm 3.89$

$40.02 \pm 4.71$

$23.82 \pm 3.54$

$32.95 \pm 3.89$

$36.31 \pm 2.78$

$39.61 \pm 2.73$

$37.98 \pm 2.85$

$25.34 \pm 4.41$

$94.67 \pm 9.37$

$134.86 \pm 12.76$

$147.28+18.80$

$155.41 \pm 16.49$

$161.20 \pm 14.43$

$96.42 \pm 9.67$

$494.96 \pm 1014.14$

$205.88+304.64$

$36.36 \pm 1466$

$286.07 \pm 569.82$

$63.71 \pm 12.82$

$58.46 \pm 5.41$

$66.65 \pm 8.34$

$10.60+3.30$

$9.10 \pm 2.84$

$10.91 \pm 3.34$

$25.41 \pm 9.44$

$20.37+8.14$

$25.58+8.94$

$37.21 \pm 6.97$

$42.97 \pm 6.04$

$37.22 \pm 7.42$
$30.68 \pm 12.30$

$120.41 \pm 33.07$

$128.26 \pm 33.78$

$128.37 \pm 33.11$

$115.91 \pm 32.15$

$32.80 \pm 8.05$

$30.19+8.28$

$116.34 \pm 24.81$

$124.55 \pm 22.01$

$128.07 \pm 22.38$

$107.20+20.07$

$31.29 \pm 10.18$

$38.22 \pm 3.82$

$35.63 \pm 3.47$

$33.78 \pm 3.98$

$30.64+3.83$

$36.76 \pm 4.21$

$40.17 \pm 2.86$

$23.18 \pm 4.35$

$32.38 \pm 3.83$

$36.40 \pm 2.56$

$39.76 \pm 2.87$

$37.66 \pm 2.46$

$25.47 \pm 3.86$

$97.99 \pm 14.90$

$34.98 \pm 11.87$

$151.99 \pm 15.26$

$150.97 \pm 14.39$

$161.80 \pm 15.00$

$100.69 \pm 9.97$

$461.57 \pm 391.46$

$213.35+176.62$

$440.06 \pm 307.87$

$210.82 \pm 190.05$

$66.91 \pm 9.33$

$59.87 \pm 5.32$

$67.67 \pm 7.91$

$10.98+3.81$

$9.56 \pm 3.31$

$11.25 \pm 3.55$

$23.98 \pm 7.85$

$18.87+6.14$

$24.26+8.18$

$38.23 \pm 8.71$

$42.93 \pm 7.44$

$37.74 \pm 8.71$ 


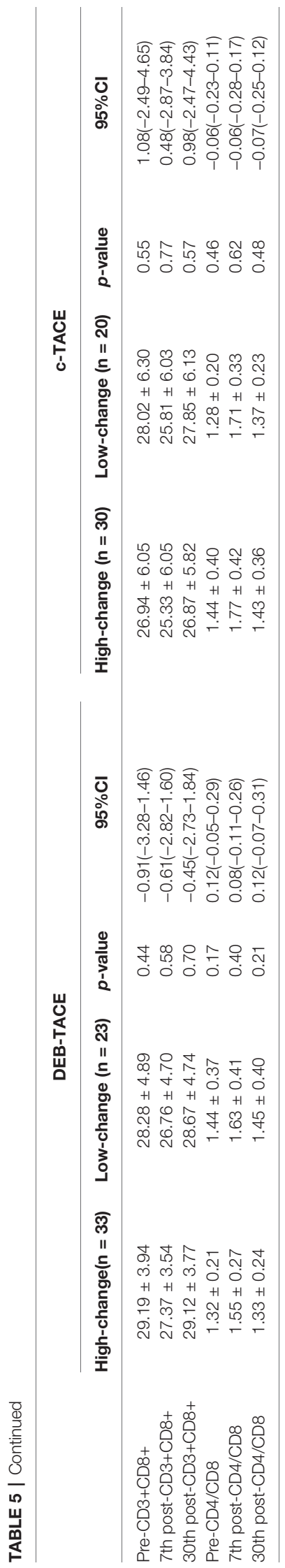

According to the statistics and analysis of common adverse reactions after TACE, patients with low pre-TACE expression of HMGB1 had a lower incidence of fever than those with higher expression. In addition, patients with a lower postoperative increase in HMGB1 expression had a lower risk of developing a fever. The results suggest that there is a correlation between the expression of HMGB1 and fever. On the one hand, post-TACE fever is due to the absorption of necrotic material at the site of embolization, which is the classic "absorption heat". On the other hand, HMGB1 can lead to the release of pro-inflammatory factors and excitatory amino acids in the microenvironment, which also promotes the development of fever in the body (35). Therefore, the high expression of HMGB1 in the serum before and after treatment may predict a higher risk of fever in patients.

DEB-TACE was modified from c-TACE. The classical c-TACE is to infuse chemotherapeutic drugs into the blood vessels of tumors, and then embolize the blood vessels with insoluble materials. The microspheres used in DEB-TACE can both adsorb drugs and serve as materials for embolization of blood vessels. Although there are some differences in the surgical procedures, the principles of the two treatments for tumors are consistent. For ischemia and hypoxia caused by c-TACE and DEB-TACE, the reperfusion injury of vascular recanalization after two TACE are consistent, and the change of HMGB1 expression level after treatment has the same trend.

During the follow-up of this subject, we found that tumor markers decreased significantly at one month after TACE. The expression changes of tumor markers in the two groups were similar, and the difference was not statistically significant, which showed that both treatment methods had good effects on liver metastasis of colorectal cancer.

Analysis of the quality of life data showed that the QoL scores increased substantially after TACE. The results show that TACE can significantly improve the quality of life.

The results of the present study showed that patients with dramatically increased HMGB1 level after TACE had a relatively poor outcome. Patients whose HMGB1 expression increased more than $50 \%$ after TACE had shorter PFS than those whose expression smaller. From the principle of TACE, the most ideal result is that the blood supply of tumors is permanently and completely blocked, combined with the killing effect of anti-tumor drugs, achieved the therapeutic purposes. However, some tumor cells survive and continue to grow after TACE due to the rapid emergence of collateral circulation and the existence of some unembolized micro-vessels. According to related studies, HMGB1 can promote the formation and development of new blood vessels (36). HMGB1 is also associated with cancer progression and immune escape, which is able to induce angiogenesis, metastasis (37). At present, HMGB1 is known to accelerate angiogenesis by 1) acting on vascular endothelium to promoting the formation of new blood vessels and neovascular network by promoting the synthesis of endothelial growth factor. 2) Acetylated HMGB1, which has been released, activates macrophages to upregulate nuclear factor kappa $\mathrm{B}$, thereby promoting the synthesis and secretion of vascular endothelial growth factor and indirectly promoting the formation of new blood vessels $(38,39)$. 3) HMGB1 can upregulate the expression of fibroblast growth factor (FGF) (40), stimulate the 

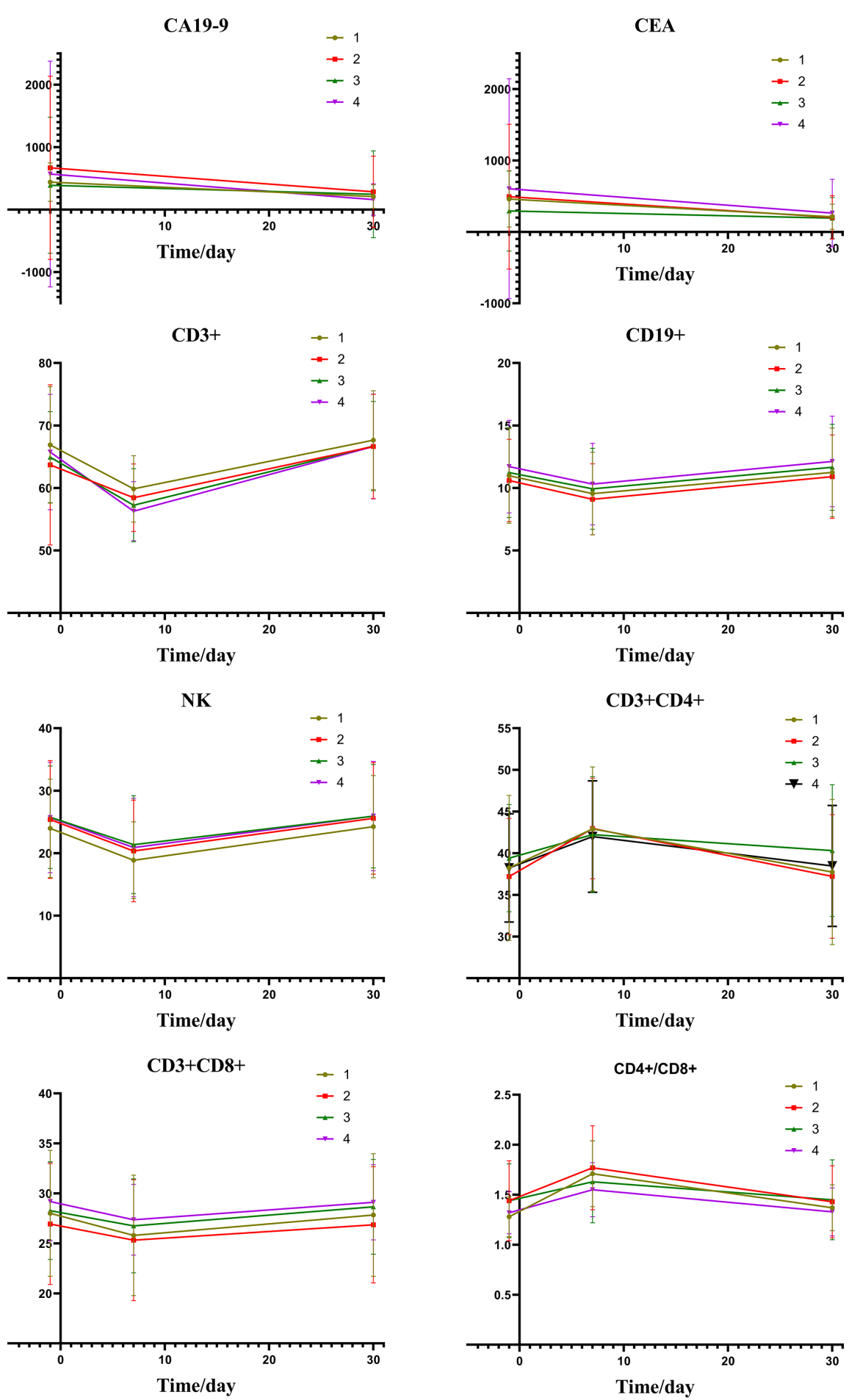

FIGURE 5 | Group1: low post-expression of HMGB1 with c-TACE; Group2: high post-expression of HMGB1 with c-TACE; Group3: low post-expression of HMGB1 with DEB-TACE; Group4: high post-expression of HMGB1 with DEB-TACE. 
TABLE 6 | Post-expression of HMGB1group, common adverse reactions and treatment outcomes.

\begin{tabular}{|c|c|c|c|c|c|c|}
\hline & \multicolumn{3}{|c|}{ DEB-TACE } & \multicolumn{3}{|c|}{ C-TACE } \\
\hline & High-change (33) & Low-change (23) & $p$-value & High-change $(30)$ & Low-change (20) & $p$-value \\
\hline fever & 18 & 6 & 0.04 & 21 & 8 & 0.03 \\
\hline vomit & 11 & 10 & 0.45 & 10 & 6 & 0.81 \\
\hline nausea & 18 & 11 & 0.63 & 11 & 12 & 0.11 \\
\hline abdominal pain & 14 & 10 & 0.94 & 10 & 8 & 0.64 \\
\hline hepatic failure & 0 & 0 & 0 & 0 & 0 & 0 \\
\hline $\mathrm{CR}^{\mathrm{a}}$ & 3 & 3 & & 2 & 2 & \\
\hline$P R^{b}$ & 13 & 17 & & 14 & 15 & \\
\hline $\mathrm{SD}^{\mathrm{c}}$ & 15 & 2 & & 12 & 2 & \\
\hline$P D^{d}$ & 2 & 1 & & 2 & 1 & \\
\hline $\mathrm{ORR}^{\mathrm{e}}$ & 16 & 20 & $<0.01$ & 16 & 17 & 0.01 \\
\hline Pre-score of Qol ${ }^{f}$ & 32.64 & 33.26 & & 31.27 & 31.50 & \\
\hline Post-score of Qol & 47.58 & 48.78 & & 48.37 & 47.70 & \\
\hline
\end{tabular}

${ }^{a} \mathrm{CR}$, complete response.

${ }^{b} \mathrm{PR}$, partial response.

${ }^{\mathrm{C}} \mathrm{SD}$, stable disease.

${ }^{d} P D$, progressive disease.

${ }^{e} \mathrm{ORR}$, Objective response rate; $\mathrm{ORR}=\mathrm{CR}+\mathrm{PR}$.

${ }^{f} \mathrm{Qol}$, quality of life.

secretion of platelet-derived growth factor (PDGF) $(41,42)$, and greatly enhance the proliferation and migration ability of endothelial cells.

Receptor for advanced glycation end products (RAGE) plays a role in tumor metastasis after binding to $\operatorname{HMGB1}(43,44)$. The C-terminus of HMGB1 can specifically bind to RAGE binding, triggering cytoplasmic signaling required for cell movement regulation and opening the molecular switches that control cytoskeletal organization (45). HMGB1/RAGE cannot only regulate the cytoskeleton to achieve cell movement, but also attract and aggregate other cells, and enhance the ability of cell aggregation and adhesion, which plays an important role in the formation of new collateral circulation after TACE. The combination of HMGB1/RAGE makes peripheral cells and smooth muscle cells aggregate to the high expression site and promotes the formation of the vascular structure. In addition, HMGB1/RAGE can regulate the expression of the BCL-2 gene (B-cell lymphoma/leukemia2 gene) $(46,47)$, which is a cancer gene with the effect of inhibiting apoptosis. The anti-apoptotic effect of HMGB1/RAGE is directly related to the expression of BCL-2 (48). The multiple effects of HMGB1 enable the tumor to rapidly establish collateral circulation after the original blood supply is interrupted by embolization, which leads to the tumor to regain some vessels after TACE. When many tumor cells are necrotic, the remaining tumor cells with blood supply at the edge grow and proliferate rapidly, which leads to the progress and recurrence of local lesions.

The small size of patients' sample in this study may have some impact on the accuracy of the results. A larger number of patients can undoubtedly increase the accuracy of the results. The results of this study need to be verified by the analysis of large samples in the future, and we hope to promote a multicenter study with a larger sample size in the next time, which can verify the predictive role of HMGB1.

Most of the patients who had better outcomes in this study are still alive. Three-year and five-year survival rates were not analyzed due to time constraints. In the future, we will continue to follow up on the enrolled patients in order to obtain complete survival data and compare their long-term survival rates.
In conclusion, the results of this study suggest that HMGB1 may serve as a marker for predicting liver injury and long-term efficacy after TACE in patients with LMCRC. The change of HMGB1 before and after TACE is significantly associated with PFS. With the help of monitoring the change of HMGB1 expression, patients' PFS can be effectively predicted.

\section{DATA AVAILABILITY STATEMENT}

The raw data supporting the conclusions of this article will be made available by the authors, without undue reservation.

\section{ETHICS STATEMENT}

The studies involving human participants were reviewed and approved by Shandong Cancer Hospital Ethics Committee. The patients/participants provided their written informed consent to participate in this study.

\section{AUTHOR CONTRIBUTIONS}

$\mathrm{J}-\mathrm{jH}$ and $\mathrm{Y}-\mathrm{dS}$ made the study concept and design. Y-qC, C-xW, and J-bZ acquired the data. HZ, J-zL and H-rX made the interpretation of data. HZ and Y-dS made the analysis and drafted the manuscript. All authors contributed to the article and approved the submitted version. $\mathrm{J}-\mathrm{jH}$ is the guarantor.

\section{FUNDING}

This work was supported by the Natural Science Foundation of Shandong Province (No. ZR2017MH095) and The Key R \& D Project of Shandong Province (No. 2019GSF108166), and Cancer Interventional Therapy Research Foundation Project of China Health Promotion Foundation (HRIRF-2018-C004). 


\section{REFERENCES}

1. Gu M, Huang Q, Bao C, Li Y, Li X, Ye D, et al. Attributable causes of colorectal cancer in China. BMC Cancer (2018) 18:38. doi: 10.1186/s12885017-3968-z

2. Maffione A, Lopci E, Bluemel C, Giammarile F, Herrmann K, Rubello D. Diagnostic accuracy and impact on management of (18)F-FDG PET and PET/CT in colorectal liver metastasis: a meta-analysis and systematic review. Eur J Nuclear Med Mol Imaging (2015) 42:152-63. doi: 10.1007/s00259-0142930-4

3. Salah S, Ardissone F, Gonzalez M, Gervaz P, Riquet M, Watanabe K, et al. Pulmonary metastasectomy in colorectal cancer patients with previously resected liver metastasis: pooled analysis. Ann Surg Oncol (2015) 22:184450. doi: 10.1245/s10434-014-4173-9

4. Ali S, Pawlik T, Rodriguez-Bigas M, Monson J, Chang G, Larson D. Timing of Surgical Resection for Curative Colorectal Cancer with Liver Metastasis. Ann Surg Oncol (2018) 25:32-7. doi: 10.1245/s10434-016-5745-7

5. R M, U S, S W, R K, H L. Incidence of synchronous liver metastases in patients with colorectal cancer in relationship to clinico-pathologic characteristics. Results of a German prospective multicentre observational study. Eur J Surg Oncol (2012) 38:259-65. doi: 10.1016/j.ejso.2011.12.013

6. Lee J, Hong H, Kim J, Lee I, Lee K, Park I, et al. Simultaneous laparoscopic resection of primary colorectal cancer and metastatic liver tumor: initial experience of single institute. J Laparoendosc Adv Surg Techn Part A (2010) 20:683-7. doi: 10.1089/lap.2010.0039

7. Yuan P, Zhang Z, Kuai J. Analysis on efficacy and safety of TACE in combination with RFA and MWA in the treatment of middle and large primary hepatic carcinoma. J B U O N (2019) 24:163-70.

8. Ma J, Wang F, Zhang W, Wang L, Yang X, Qian Y, et al. Percutaneous cryoablation for the treatment of liver cancer at special sites: an assessment of efficacy and safety. Quant Imaging Med Surg (2019) 9:1948-57. doi: 10.21037/ qims.2019.11.12

9. JC C, EN P, CT S. Thermal Ablation of Metastatic Colon Cancer to the Liver. Semin Intervent Radiol (2019) 36:310-8. doi: 10.1055/s-0039-1698754

10. M L, A S, S R. Collagen Kinase Receptors as Potential Therapeutic Targets in Metastatic Colon Cancer. Front Oncol (2020) 10:125. doi: 10.3389/ fonc. 2020.00125

11. A F, M G, J B, JL R. Treatment of intermediate-stage hepatocellular carcinoma. Nature reviews. Clin Oncol (2014) 11:525-35. doi: 10.1038/ nrclinonc.2014.122

12. Yang $\mathrm{Q}$, Jin $\mathrm{X}$, Ye F, Zheng B, Xie X, Luo Y, et al. Safety and efficacy analysis of DEB-TACE treatment in elderly patients with hepatocellular carcinoma: a comparative cohort study. Oncol Res (2018). doi: 10.3727/096504018x1522 3171140640

13. Jilin Y, Xiaofeng L, Zhifang Y, Xingrui L. Study on the changes in microvessel density in hepatocellular carcinoma following transcatheter arterial chemoembolization. J Tongji Med Univ (2001) 21:321-2. doi: 10.1007/ BF02886568

14. Guiu B, Deschamps F, Aho S, Munck F, Dromain C, Boige V, et al. Liver/ biliary injuries following chemoembolisation of endocrine tumours and hepatocellular carcinoma: lipiodol vs. drug-eluting beads. J Hepatol (2012) 56:609-17. doi: 10.1016/j.jhep.2011.09.012

15. RA M, S O, F X, M F, F P. Liver function changes after transarterial chemoembolization in US hepatocellular carcinoma patients: the LiverT study. BMC Cancer (2019) 19:795. doi: 10.1186/s12885-019-5989-2

16. Nakamura K, Kageyama S, Kaldas F, Hirao H, Ito T, Kadono K, et al. Hepatic CEACAM1 expression indicates donor liver quality and prevents early transplantation injury. J Clin Invest (2020) 130:2689-704. doi: 10.1172/ jci133142

17. N K, D N, D J, P S, S H. Predictive value of immunogenic cell death biomarkers HMGB1, sRAGE, and DNase in liver cancer patients receiving transarterial chemoembolization therapy. Tumour Biol (2012) 33:2401-9. doi: 10.1007/s13277-012-0504-2

18. Sosa R, Terry A, Kaldas F, Jin Y, Rossetti M, Ito T, et al. Disulfide-HMGB1 Drives Ischemia-Reperfusion Injury in Human Liver Transplantation. Hepatol (Baltimore Md) (2020). doi: 10.1002/hep.31324

19. Napolitano A, Antoine D, Pellegrini L, Baumann F, Pagano I, Pastorino S, et al. HMGB1 and Its Hyperacetylated Isoform are Sensitive and Specific
Serum Biomarkers to Detect Asbestos Exposure and to Identify Mesothelioma Patients. Clin Cancer Res (2016) 22:3087-96. doi: 10.1158/1078-0432.ccr-151130

20. Y W, Z J, J Y, S Y. HMGB1 as a Potential Biomarker and Therapeutic Target for Malignant Mesothelioma. Dis Markers (2019) 2019:4183157. doi: 10.1155/ $2019 / 4183157$

21. Venereau E, De Leo F, Mezzapelle R, Careccia G, Musco G, Bianchi M. HMGB1 as biomarker and drug target. Pharmacol Res (2016) 111:534-44. doi: 10.1016/j.phrs.2016.06.031

22. Tirone M, Tran N, Ceriotti C, Gorzanelli A, Canepari M, Bottinelli R, et al. High mobility group box 1 orchestrates tissue regeneration via CXCR4. J Exp Med (2018) 215:303-18. doi: 10.1084/jem.20160217

23. Yao X, Zhao G, Yang H, Hong X, Bie L, Liu G. Overexpression of highmobility group box 1 correlates with tumor progression and poor prognosis in human colorectal carcinoma. J Cancer Res Clin Oncol (2010) 136:677-84. doi: 10.1007/s00432-009-0706-1

24. T H, H A, S K. Balloon-occluded transcatheter arterial chemoembolization for hepatocellular carcinoma. World J Hepatol (2018) 10:485-95. doi: 10.4254/ wjh.v10.i7.485

25. AP B, AA B, SV V, II G. Molecular mechanisms of oxidation damage and liver cell dysfunction in patients with metastatic colorectal cancer. Exp Oncol (2019) 41:328-34. doi: 10.32471/exp-oncology.2312-8852.vol-41-no4.13796

26. Fang $P$, Liang J, Jiang $X$, Fang $X$, Wu M, Wei X, et al. viaQuercetin Attenuates d-GaLN-Induced L02 Cell Damage by Suppressing Oxidative Stress and Mitochondrial Apoptosis Inhibition of HMGB1. Front Pharmacol (2020) 11:608. doi: 10.3389/fphar.2020.00608

27. Huang S, Wang S, Chang S, Chuang K, Wang H, Kao J, et al. Imiquimod Exerts Antitumor Effects by Inducing Immunogenic Cell Death and Is Enhanced by the Glycolytic Inhibitor 2-Deoxyglucose. I Invest Dermatol (2020) 140:1771-83.e6. doi: 10.1016/j.jid.2019.12.039

28. Hua S, Ma M, Fei X, Zhang Y, Gong F, Fang M. Glycyrrhizin attenuates hepatic ischemia-reperfusion injury by suppressing HMGB1-dependent GSDMD-mediated kupffer cells pyroptosis. Int Immunopharmacol (2019) 68:145-55. doi: 10.1016/j.intimp.2019.01.002

29. S A, Y U, K T, S O, T Y. Kupffer cell-mediated exacerbation of methimazoleinduced acute liver injury in rats. J Appl Toxicol JAT (2016) 36:702-15. doi: 10.1002 /jat.3202

30. Zhang C, Mo M, Ding W, Liu W, Yan D, Deng J, et al. High-mobility group box 1 (HMGB1) impaired cardiac excitation-contraction coupling by enhancing the sarcoplasmic reticulum (SR) $\mathrm{Ca}(2+)$ leak through TLR4-ROS signaling in cardiomyocytes. J Mol Cell Cardiol (2014) 74:260-73. doi: 10.1016/j.yjmcc.2014.06.003

31. Lv Y, Li Y, Zhang D, Zhang A, Guo W, Zhu S. HMGB1-induced asthmatic airway inflammation through GRP75-mediated enhancement of ERmitochondrial Ca transfer and ROS increased. J Cell Biochem (2018) 119:4205-15. doi: 10.1002/jcb.26653

32. Lemaire F, Sigrist S, Delpy E, Cherfan J, Peronet C, Zal F, et al. Beneficial effects of the novel marine oxygen carrier M101 during cold preservation of rat and human pancreas. J Cell Mol Med (2019) 23:8025-34. doi: 10.1111/ jcmm.14666

33. Porto A, Palumbo R, Pieroni M, Aprigliano G, Chiesa R, Sanvito F, et al. Smooth muscle cells in human atherosclerotic plaques secrete and proliferate in response to high mobility group box 1 protein. FASEB J (2006) 20:2565-6. doi: 10.1096/fj.06-5867fje

34. Li R, Shang Y, Yu Y, Zhou T, Xiong W, Zou X. High-mobility group box 1 protein participates in acute lung injury by activating protein kinase $\mathrm{R}$ and inducing M1 polarization. Life Sci (2020) 246:117415. doi: 10.1016/ j.lfs. 2020.117415

35. Wang S, Cai S, Zhang W, Liu X, Li Y, Zhang C, et al. High-mobility group box 1 protein antagonizes the immunosuppressive capacity and therapeutic effect of mesenchymal stem cells in acute kidney injury. J Trans Med (2020) 18:175. doi: 10.1186/s12967-020-02334-8

36. W D, J Z, R Z, J Z. Curcumin inhibits the lymphangiogenesis of gastric cancer cells by inhibiton of HMGB1/VEGF-D signaling. Int J Immunopathol Pharmacol (2019) 33:2058738419861600. doi: 10.1177/2058738419861600

37. Kang R, Chen R, Zhang Q, Hou W, Wu S, Cao L, et al. HMGB1 in health and disease. Mol Aspects Med (2014) 40:1-116. doi: 10.1016/j.mam.2014.05.001 
38. Song Y, Zou X, Zhang D, Liu S, Duan Z, Liu L. Self-enforcing HMGB1/NF$\kappa B / H I F-1 \alpha$ Feedback Loop Promotes Cisplatin Resistance in Hepatocellular Carcinoma Cells. J Cancer (2020) 11:3893-902. doi: 10.7150/jca.42944

39. Alzokaky A, Abdelkader E, El-Dessouki A, Khaleel S, Raslan N. Cphycocyanin protects against ethanol-induced gastric ulcers in rats: Role of

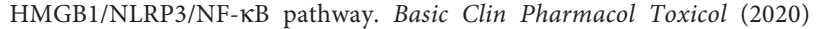
127:265-77. doi: 10.1111/bcpt.13415

40. L Z, Y J, Z K, C L, W J. Protocatechuic aldehyde ameliorates experimental pulmonary fibrosis by modulating HMGB1/RAGE pathway. Toxicol Appl Pharmacol (2015) 283:50-6. doi: 10.1016/j.taap.2015.01.001

41. He W, Xu F, Chen L, Huang W, Jiang L, Tang F, et al. Association of HighMobility Group Box-1 with Inflammation-Related Cytokines in the Aqueous Humor with Acute Primary Angle-Closure eyes. Curr Mol Med (2020). doi: 10.2174/1566524020666200413113107

42. Suwinski R, Giglok M, Galwas-Kliber K, Idasiak A, Jochymek B, Deja R, et al. Blood serum proteins as biomarkers for prediction of survival, locoregional control and distant metastasis rate in radiotherapy and radio-chemotherapy for non-small cell lung cancer. BMC Cancer (2019) 19:427. doi: 10.1186/ s12885-019-5617-1

43. GP S, DC R, ST R, R H, AJ C. HMGB1 and RAGE in inflammation and cancer. Annu Rev Immunol (2010) 28:367-88. doi: 10.1146/annurev.immunol. 021908.132603

44. Andrassy M, Volz H, Igwe J, Funke B, Eichberger S, Kaya Z, et al. Highmobility group box-1 in ischemia-reperfusion injury of the heart. Circulation (2008) 117:3216-26. doi: 10.1161/circulationaha.108.769331
45. Thakur V, Sadanandan J, Chattopadhyay M. High-Mobility Group Box 1 Protein Signaling in Painful Diabetic Neuropathy. Int J Mol Sci (2020) 21. doi: 10.3390/ijms21030881

46. Ni Z, Dai X, Wang B, Ding W, Cheng P, Xu L, et al. Natural Bcl-2 inhibitor (-)- gossypol induces protective autophagy via reactive oxygen species-high mobility group box 1 pathway in Burkitt lymphoma. Leuk Lymphoma (2013) 54:2263-8. doi: 10.3109/10428194.2013.775437

47. R K, KM L, HJ Z, MT L, D T. HMGB1: a novel Beclin 1-binding protein active in autophagy. Autophagy (2010) 6:1209-11. doi: 10.4161/auto.6.8.13651

48. SA W, W W, AK B, HS E-A. Galantamine anti-colitic effect: Role of alpha-7 nicotinic acetylcholine receptor in modulating Jak/STAT3, NF- $\mathrm{KB} / \mathrm{HMGB1} /$ RAGE and p-AKT/Bcl-2 pathways. Sci Rep (2018) 8:5110. doi: 10.1038/ s41598-018-23359-6

Conflict of Interest: The authors declare that the research was conducted in the absence of any commercial or financial relationships that could be construed as a potential conflict of interest.

Copyright (c) 2020 Sun, Zhang, Chen, Wu, Zhang, Xu, Liu and Han. This is an openaccess article distributed under the terms of the Creative Commons Attribution License (CC BY). The use, distribution or reproduction in other forums is permitted, provided the original author(s) and the copyright owner(s) are credited and that the original publication in this journal is cited, in accordance with accepted academic practice. No use, distribution or reproduction is permitted which does not comply with these terms. 